\title{
Spin-Orbital Quantum Liquid on the Honeycomb Lattice
}

\author{
Philippe Corboz, ${ }^{1}$ Miklós Lajkó, ${ }^{2,3}$ Andreas M. Läuchli, ${ }^{4}$ Karlo Penc, ${ }^{2,3}$ and Frédéric Mila ${ }^{5}$ \\ ${ }^{1}$ Theoretische Physik, ETH Zurich, 8093 Zurich, Switzerland \\ ${ }^{2}$ Institute for Solid State Physics and Optics, Wigner Research Centre for Physics, Hungarian Academy of Sciences, \\ Post Office Box 49, H-1525 Budapest, Hungary \\ ${ }^{3}$ Department of Physics, Budapest University of Technology and Economics, 1111 Budapest, Hungary \\ ${ }^{4}$ Institut für Theoretische Physik, Universität Innsbruck, A-6020 Innsbruck, Austria \\ ${ }^{5}$ Institut de théorie des phénomènes physiques, École Polytechnique Fédérale de Lausanne (EPFL), CH-1015 Lausanne, Switzerland
}

(Received 27 July 2012; published 27 November 2012)

\begin{abstract}
The main characteristic of Mott insulators, as compared to band insulators, is to host low-energy spin fluctuations. In addition, Mott insulators often possess orbital degrees of freedom when crystal-field levels are partially filled. While in the majority of Mott insulators, spins and orbitals develop long-range order, the possibility for the ground state to be a quantum liquid opens new perspectives. In this paper, we provide clear evidence that the spin-orbital SU(4) symmetric Kugel-Khomskii model of Mott insulators on the honeycomb lattice is a quantum spin-orbital liquid. The absence of any form of symmetry breakinglattice or $\mathrm{SU}(\mathrm{N})$ - is supported by a combination of semiclassical and numerical approaches: flavor-wave theory, tensor network algorithm, and exact diagonalizations. In addition, all properties revealed by these methods are very accurately accounted for by a projected variational wave function based on the $\pi$-flux state of fermions on the honeycomb lattice at $1 / 4$ filling. In that state, correlations are algebraic because of the presence of a Dirac point at the Fermi level, suggesting that the symmetric Kugel-Khomskii model on the honeycomb lattice is an algebraic quantum spin-orbital liquid. This model provides an interesting starting point to understanding the recently discovered spin-orbital-liquid behavior of $\mathrm{Ba}_{3} \mathrm{CuSb}_{2} \mathrm{O}_{9}$. The present results also suggest the choice of optical lattices with honeycomb geometry in the search for quantum liquids in ultracold four-color fermionic atoms.
\end{abstract}

DOI: 10.1103/PhysRevX.2.041013

Subject Areas: Computational Physics, Condensed Matter Physics, Strongly Correlated Materials

\section{INTRODUCTION}

The investigation of orbital physics in transition-metal oxides has recently been boosted by the possibility of observing orbital excitations with resonant inelastic x-ray scattering [1]. This possibility has been demonstrated in cases where the crystal-field splitting is strong enough to select a unique orbital configuration in the ground state and push the orbital excitations to high energy and hence to separate them from magnetic excitations. However, such a separation of orbital and magnetic excitations is not the only possibility. If the electronic configuration of the transition-metal ion is such that several orbital occupations are consistent with the crystal-field environment, a situation referred to as orbital degeneracy [2], orbital fluctuations are expected to have an energy comparable to that of spin fluctuations. In most cases known until recently, a cooperative Jahn-Teller distortion occurs, resulting in orbital order and gapped orbital excitations, but there is no reason for such a distortion to take place $a$ priori, and the search for situations in which orbitals remain fluctuating in

Published by the American Physical Society under the terms of the Creative Commons Attribution 3.0 License. Further distribution of this work must maintain attribution to the author( $s)$ and the published article's title, journal citation, and DOI. the ground state has been very active over the past decade [3-9]. To which extent the orbitals keep fluctuating in the ground state of the triangular system $\mathrm{LiNiO}_{2}[10,11]$ or in the spinel $\mathrm{FeSc}_{2} \mathrm{~S}_{4}$ [12] is still debated [13]. Interestingly, a new candidate has recently been put forward, $\mathrm{Ba}_{3} \mathrm{CuSb}_{2} \mathrm{O}_{9}$ [14], a $\mathrm{Cu}$ oxide that lives on a decorated honeycomb lattice in which no trace of orbital order could be detected.

On the theory side, the Mott-insulating state in transition-metal oxides with orbital degeneracy is generally described by a Kugel-Khomskii model [15] in which spin and orbital degrees of freedom are coupled on each bond. A minimal model to investigate the possibility of stabilizing an orbital liquid is the symmetric version of that model defined by the Hamiltonian

$$
\mathcal{H}=\sum_{\langle i, j\rangle}\left(2 \mathbf{S}_{i} \cdot \mathbf{S}_{j}+\frac{1}{2}\right)\left(2 \mathbf{T}_{i} \cdot \mathbf{T}_{j}+\frac{1}{2}\right),
$$

where the sum goes over nearest-neighbor sites, $\mathbf{S}_{i}$ is a spin- $1 / 2$ operator, and $\mathbf{T}_{i}$ is a pseudospin- $1 / 2$ operator that describes fluctuations of a two-fold degenerate orbital ( $a$ and $b)$. Introducing the local basis $|\bullet\rangle=|\uparrow a\rangle,|\bullet\rangle=|\downarrow a\rangle,|\bullet\rangle=|\uparrow b\rangle,|\ominus\rangle=|\downarrow b\rangle$, the Hamiltonian exhibits the full SU(4) symmetry and can be written as $\mathcal{H}=\sum_{\langle i, j\rangle} P_{i, j}$, where $P_{i, j}$ interchanges the states on sites $i$ and $j$. The local basis states are often referred to as 
colors. In fact, the model is the straightforward generalization of the $\mathrm{SU}(2)$ symmetric Heisenberg model for $S=1 / 2$ spins which, up to a constant and a factor 2 , has the same form when expressed with $P_{i, j}$ operators.

The first investigations of this model on various lattices have emphasized the role of four-site plaquettes, the natural unit to use to build an $\mathrm{SU}(4)$ singlet $[5,16,17]$. The spontaneous formation of four-site plaquettes has been proven for an SU(4) ladder [17], and plaquette coverings have been argued to provide the relevant variational subspace for the ground-state properties of the SU(4) model on both the square and triangular lattices $[5,16]$, with possibly plaquette long-range order on the square lattice $[18,19]$. In a variational study based on projected fermionic wave functions, a gapless spinorbital-liquid state has been predicted in Ref. [8] on the square lattice. These previous conclusions have recently been challenged for the square lattice [20], for which spontaneous dimerization (as opposed to tetramerization) has been demonstrated on the basis of state-of-the-art infinite projected entangled-pair state (iPEPS) simulations. In the dimerized phase, the dimers are antisymmetric states built out of two of the four colors, and they are arranged into a columnar pattern in which each dimer has a neighboring dimer above itself and another one below. Each dimer is preferentially surrounded by dimers built out of the other two colors, leading to long-range color order [20]. In the context of orbital degeneracy, this phase is likely to be ordered. Indeed, one of the states selected by the dimerization consists of alternating pairs of $a$ and $b$ orbitals times a spin singlet. When coupled to the lattice, such a state is expected to undergo a cooperative Jahn-Teller distortion that will stabilize orbitals $a$ and $b$ and hence to lead to long-range orbital order.
In this paper, we consider the symmetric KugelKhomskii model on the honeycomb lattice. The first motivation is purely theoretical: Since there are no foursite plaquettes on this lattice, the ground state is unlikely to be a crystal of singlet plaquettes. The second motivation comes from experiments: The recent observation of a spinliquid behavior in $\mathrm{Ba}_{3} \mathrm{CuSb}_{2} \mathrm{O}_{9}$ points to the honeycomb geometry as an outstanding candidate.

The main result of the present investigation is summarized in Fig. 1. The SU(4) symmetric Kugel-Khomskii model is shown to be a quantum spin-orbital liquid with short-range color correlations that follow the pattern illustrated in Fig. 1(a), and strong evidence is provided in favor of an algebraic spin-orbital liquid with typical conical singularities in the static structure factor, as shown in Fig. 1(b).

To reach these conclusions, we have used a variety of analytical and numerical methods: linear flavor-wave theory (LFWT), iPEPS, exact diagonalization of finite clusters (ED), and a variational approach based on the Monte Carlo sampling (VMC) of Gutzwiller projected fermionic wave functions. Details about each method are found in Appendix A. These methods are complementary and shed light on different aspects of the model: LFWT is a good starting point to test for lattice symmetry breaking and color order. iPEPS is a variational approach for infinite systems that has proven to be very successful for checking the presence of any kind of long-range order $[20,21]$. Exact diagonalizations reveal nearly exact information on short-length-scale properties and are extremely useful for benchmarking other approaches. The variational Monte Carlo sampling of fermionic wave functions has proven to provide a remarkably accurate description of algebraic quantum liquids. As a first test, we compare in
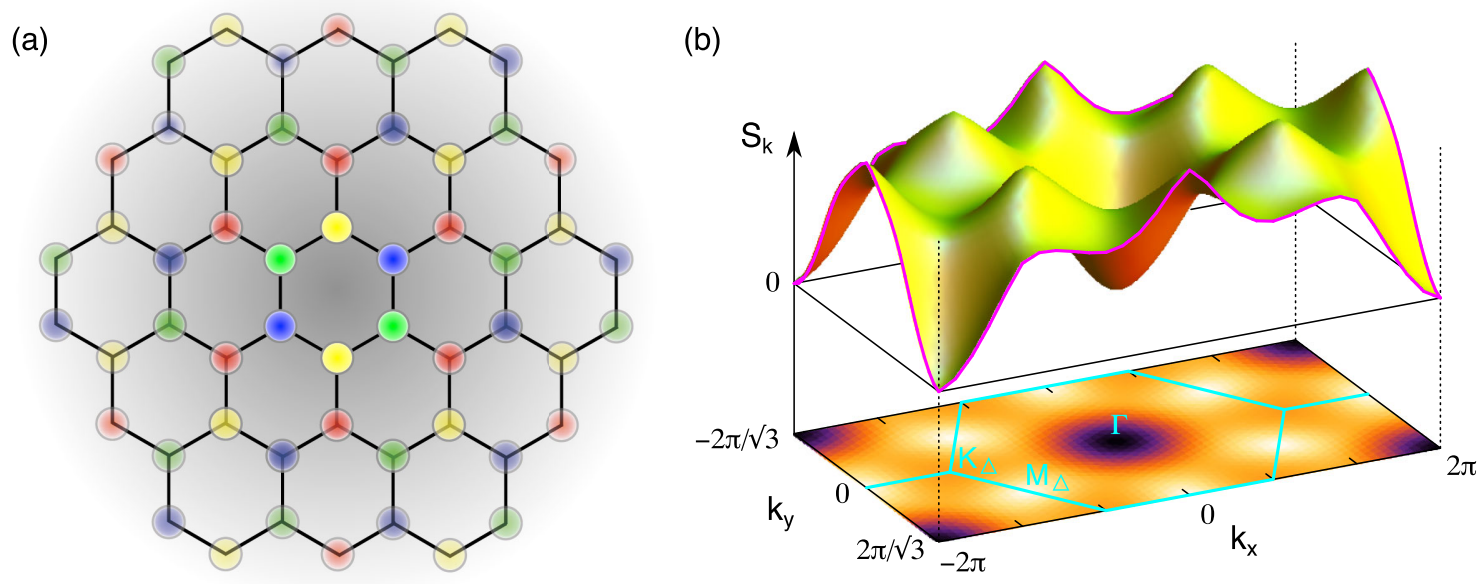

FIG. 1. Summary of the main properties of the spin-orbital liquid of the SU(4) model on the honeycomb lattice. (a) Sketch of local color order. (The fading of colors illustrates the absence of long-range color order.) This pattern is the only one that respects the sequence of four colors along all the zigzag chains, whatever their orientation (horizontal, $\pi / 3$, or $2 \pi / 3$ ). (b) Color-structure factor of the Gutzwiller projected $\pi$-flux state (VMC). The singular conical peaks are typical of algebraic correlations. 


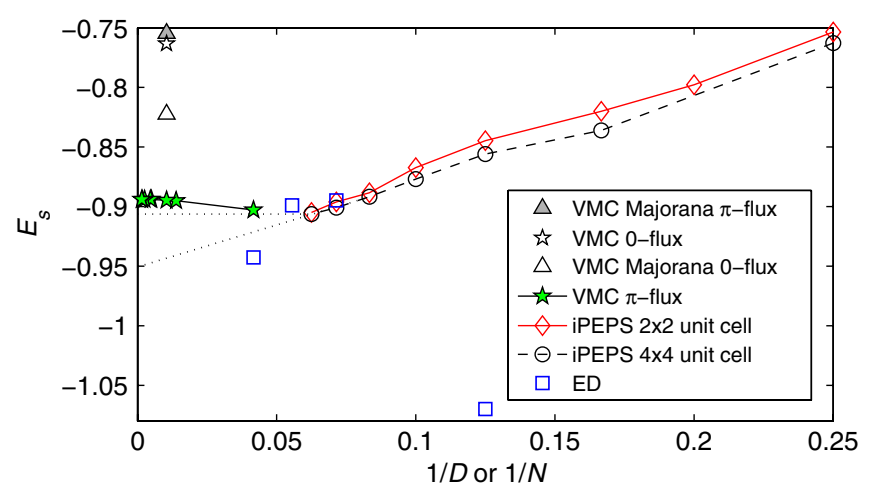

FIG. 2. Energy per site as a function of inverse bond dimension $D$ (iPEPS) and as a function of inverse system size $N$ (VMC and ED). We note that the LFWT energy $\left(E_{s}=-1.5\right.$, not shown) is not variational.

Fig. 2 the ground-state energies of the various approaches. A number of conclusions can already be drawn from this comparison. First, among all the Gutzwiller projected fermionic wave functions we have considered, only one is really competitive: the wave function based on the quarter-filled Fermi sea of standard fermions in the $\pi$-flux state (see Sec. II C for details). Its energy is much lower than that of the 0 -flux state, as well as that of the halffilled Fermi sea of Majorana fermions with 0 or $\pi$ flux, and these alternative fermionic wave functions are not considered any further [22]. Second, the agreement between iPEPS, ED, and the Gutzwiller projected $\pi$-flux state is quite remarkable, which suggests that all these methods constitute appropriate descriptions within their range of validity [23].

\section{SIMULATION RESULTS}

\section{A. Absence of lattice symmetry breaking}

Lattice symmetry breaking, be it dimerization or plaquette formation, leads to bonds of different strengths. At the classical level, which consists of minimizing the energy in the subspace of product wave functions of the form $|\psi\rangle=\prod_{i}\left|\psi_{i}\right\rangle$, all bonds are fully satisfied. Indeed, since the Hamiltonian of a bond $H_{i j}$ is a simple permutation, $\left\langle\psi_{i} \psi_{j}\left|H_{i j}\right| \psi_{i} \psi_{j}\right\rangle=\left|\left\langle\psi_{i} \mid \psi_{j}\right\rangle\right|^{2}$ is minimal if neighboring states are orthogonal. On bipartite lattices such as the square or honeycomb lattices, it takes only two colors to achieve orthogonality, and the classical ground state for more than two colors is massively degenerate. This degeneracy, however, can in principle be lifted by zeropoint fluctuations. The theory of harmonic fluctuations has been developed previously; it is called flavor-wave theory. (See Appendix A 1 for details.) At the harmonic level, the energy of a bond takes the smallest possible value if the two colors of the bond are different from colors of all the other sites directly connected to the bod, i.e., of all the other sites that are nearest neighbors to one of the sites of the bond. For the honeycomb lattice, this condition can be fulfilled for all bonds simultaneously in an infinite number of ways. The configuration of Fig. 1(a) is the most symmetric one. In this configuration, all bonds have the same surrounding up to color permutations. Other configurations can be generated by exchanging the colors on a stripe of dimers [see Figs. 3(a) and 3(b)], leading to a degeneracy of order $2^{\sqrt{N}}$ since, once a direction has been chosen, this exchange can be done independently on all dimer stripes. In all configurations, the energy is the same on all bonds. This is a first hint that, by contrast to the square lattice, the lattice symmetry is not broken for the honeycomb lattice.

The same family of degenerate ground states is obtained with iPEPS if a unit cell with $4 \times 4=16$ different tensors and a small bond dimension $D=2$ are used. Upon increasing $D$, more quantum fluctuations are taken into account, and the symmetric state of Fig. 3(a) is stabilized. In this state, all bonds have the same energy [see Fig. 3(c)]. To test how robust this conclusion is, we have challenged it by performing iPEPS simulations using a $2 \times 2$ unit cell with only four different tensors. These simulations lead to a dimerized state with two types of dimers, A and B, which can be distinguished by their dominant colors, and
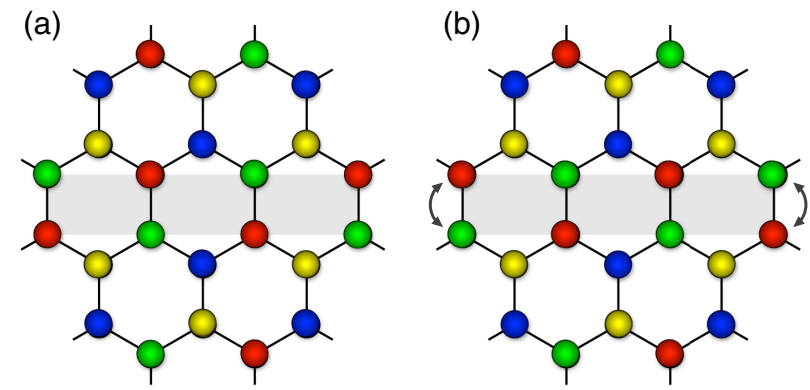

(c)

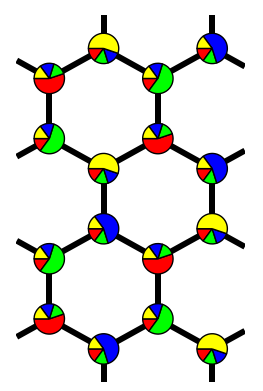

(d)

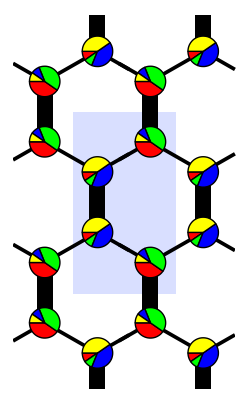

FIG. 3. Examples of states obtained with LFWT (a),(b) and iPEPS for small bond dimension $D$ (c),(d). (a) Most symmetric configuration. (b) Configuration obtained from the most symmetric one by exchanging colors on a stripe. (c) Color-ordered state with one dominant color per site, obtained with a $4 \times 4$ unit cell and a bond dimension $D=6$. (d) Dimer-Néel ordered state obtained with a $2 \times 2$ unit cell (shaded in blue) and $D=6$. Both the color order and the dimerization vanish in the infinite- $D$ limit. The pie charts show the local color density on each site, and the thickness of a bond is proportional to the square of the energy on the corresponding bond. 
different inter- and intradimer bond energies [Fig. 3(d)]. However, unlike on the square lattice, this dimerization vanishes in the infinite- $D$ limit, as shown in Fig. 4(a) where the difference in bond energies, $\Delta E_{b}=\max \left(E_{b}\right)-$ $\min \left(E_{b}\right)$, is plotted. Thus, both low-energy states found with iPEPS preserve the lattice symmetry in the large- $D$ limit.

We have also tested with VMC the stability of the $\pi$-flux state toward the dimerization instability shown in Fig. 3(d) by strengthening and weakening the hopping amplitude of the bonds, with the conclusion that the variational energy is minimal in the absence of any dimerization. Similarly, we have found no indication toward quadrumerization [SU(4) singlet formation], where the hoppings connected to, say, red sites (forming a "tripod") in Fig. 1(a) are modified.

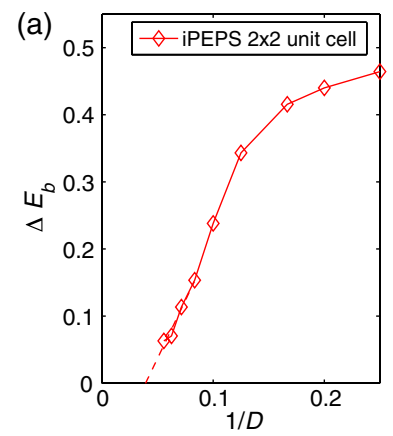

(b)

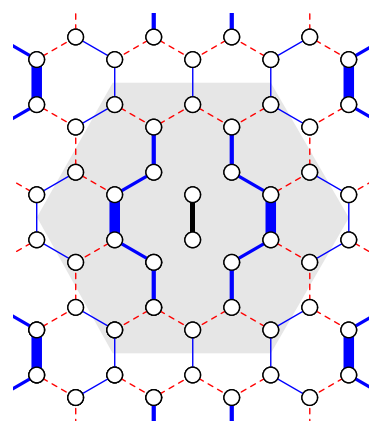

(c)
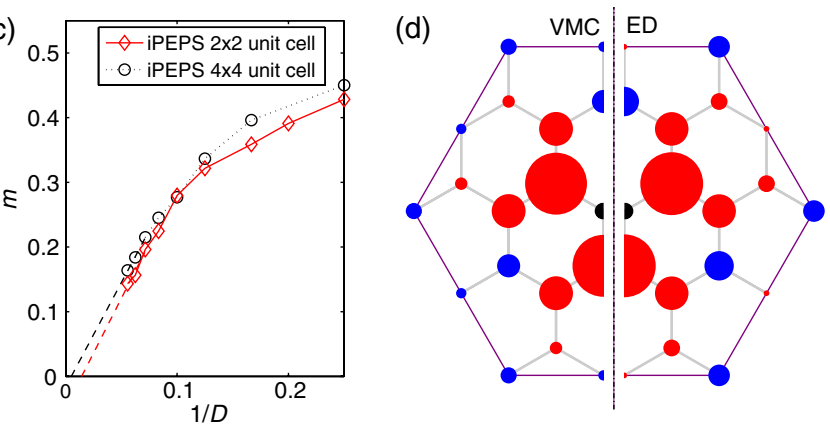

FIG. 4. Various correlation functions obtained with iPEPS, ED, and VMC methods. (a) Difference in bond energies obtained with iPEPS in the state shown in Fig. 3(d). The difference is strongly suppressed with increasing bond dimension $D$ and vanishes in the infinite- $D$ limit. (b) Connected bond-energy correlations $\left\langle P_{i j} P_{k l}\right\rangle-\left\langle P_{i j}\right\rangle\left\langle P_{k l}\right\rangle$ calculated with ED in the ground state of the $N=24$ sample. The black bonds denote the reference bond. Solid blue (dashed red) bonds stand for positive (negative) correlation functions, and the width of the bond is proportional to the absolute value of the correlation function. (c) Local ordered moment $m$ obtained with iPEPS as a function of inverse bond dimension. The moment vanishes in the infinite- $D$ limit for both low-energy states shown in Figs. 3(c) and 3(d). (d) Spin-correlation function in real space, as calculated from ED (right) and VMC (left) for a 24-site cluster. The area is proportional to $\left\langle P_{0 i}\right\rangle-1 / 4$, where 0 is the index of the central site, and $i$ labels the sites in the 24 -site cluster. The color keeps track of the sign (blue for positive, red for negative).
Finally, in Fig. 4(b), we show the ED results for the connected bond-energy correlations (described in the caption), which provide a way to detect dimerization and tendencies toward the formation of other bond-energy patterns. The correlations decay quite rapidly with distance, making dimerization or other patterns unlikely.

Thus, all methods consistently point toward a state that does not break the lattice symmetry.

\section{B. Absence of SU(4) symmetry breaking}

The color-ordered states predicted by LFWT and iPEPS with a small bond dimension (Fig. 3) break the SU(4) symmetry. Here, we show that higher-order quantum fluctuations destroy this color order, i.e., that in the ground state the SU(4) symmetry is in fact not broken.

In Fig. 4(c), we present the iPEPS result for the local ordered moment,

$$
m=\sqrt{\frac{4}{3} \sum_{\alpha, \beta}\left(\left\langle S_{\alpha}^{\beta}\right\rangle-\frac{\delta_{\alpha \beta}}{4}\right)^{2}},
$$

where $S_{\alpha}^{\beta}=|\alpha\rangle\langle\beta|$ are the generators of SU(4) and $\alpha, \beta$ run over the four different flavors. A finite $m$ implies that the SU(4) symmetry is spontaneously broken in the thermodynamic limit. For both low-energy states found with iPEPS, the local ordered moment is strongly suppressed with increasing bond dimension, and most likely vanishes in the large- $D$ limit: Quantum fluctuations, which are systematically taken into account by increasing $D$, eventually destroy the color order so that the SU(4) symmetry is restored. The situation is different on the square lattice [20], where the local ordered moment has been found to remain finite in the infinite- $D$ limit.

Consistent results for the flavor-correlation function are obtained with ED and VMC for the $\pi$-flux state shown in Fig. 4(d), which decays rapidly with increasing distance, indicating absence of long-range order. The very good qualitative and quantitative agreement between the ED and the VMC results provides substantial evidence that the $\pi$-flux state correctly describes the short-range physics of the ground state of the Hamiltonian (1). In the next section, we show that the decay predicted by VMC is algebraic, i.e., that the state described by this wave function is an algebraic spin-orbital liquid.

\section{Algebraic spin-orbital liquid}

A standard way to describe spin liquids for SU(2) models is based on the fermionic representation of the spin operators [24-28] using a variational wave function $[29,30]$, where the multiply occupied sites are projected out from a suitable chosen, noninteracting Fermi sea. While in the generic case, the Fermi sea has a finite Fermi surface, there are also other possibilities. For the SU(2) Heisenberg model on the square lattice, Affleck and Marston have shown that introducing a $\pi$ flux per 
elementary plaquette leads to the formation of Dirac nodes [31]. At half filling, the Fermi surface of this $\pi$-flux state shrinks to points, and its energy is lower than that of the state with equal hopping amplitudes and a finite Fermi surface. In such a spin liquid, the structure factor is singular at momenta related to the difference between Fermi points, leading to the algebraic decay of spin correlations. In one dimension, this type of approach leads to an accurate description of the algebraic decay of the correlations for the $\mathrm{SU}(2)$ case [32], and for $\mathrm{SU}(4)$ as well, using the representation $S_{\alpha}^{\beta}=f_{\alpha}^{\dagger} f_{\beta}[8]$.

On the honeycomb lattice, a Dirac node is already present at the middle of the band without any flux, and the Fermi surface reduces to points at half filling. So the 0 -flux state would be a good starting point to describe an algebraic spin liquid for the $\mathrm{SU}(2)$ Heisenberg model [33-35]. However, for the SU(4) Heisenberg model, the band must be quarter-filled, and the equivalent of the Affleck-Marston approach requires one to have a Dirac node at the Fermi energy of the quarter-filled system. It turns out that these properties are realized in the $\pi$-flux state, as shown in Fig. 5. As for the square lattice, this state (a)

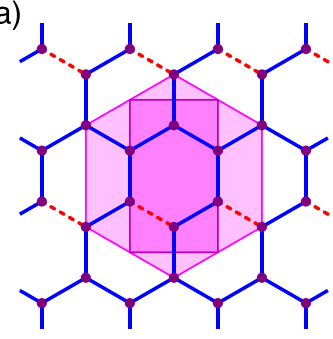

(b)

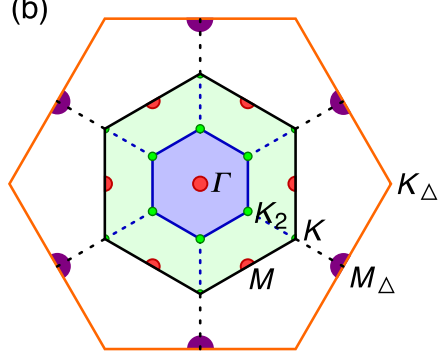

(c)

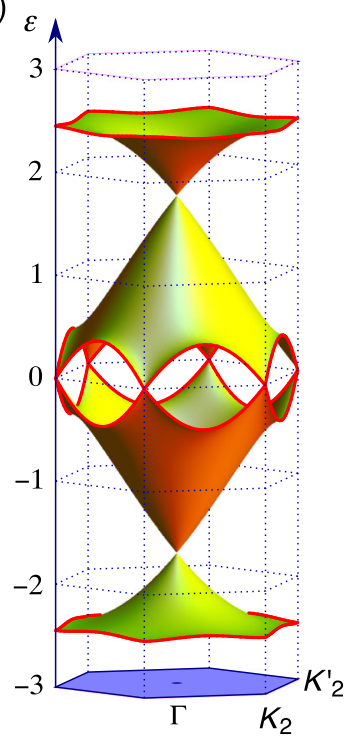

FIG. 5. Properties of the $\pi$-flux state. (a) Sketch of the gauge used to implement the $\pi$-flux state: the hopping amplitude is positive on solid blue bonds, negative on dashed red bonds. The primitive unit cell (dark magenta) contains four sites, the hexagonal unit cell eight sites. (b) Brillouin zones and high symmetry points. The red circles indicate the position of the Dirac nodes at $\varepsilon_{D}= \pm \sqrt{3} t$ to which the Fermi surface reduces at quarter filling in the $\pi$-flux state. The orange, outermost hexagon shows the extended Brillouin zone of the triangular lattice (including sites at the centers of the hexagons in the honeycomb lattice), the structure factor is maximal and has a cusp at $M_{\triangle}=$ $(\pi, \pi / \sqrt{3})$ and the symmetry related points. $K_{\triangle}$ is given by $(4 \pi / 3,0), K$ is $(2 \pi / 3,2 \pi / 3 \sqrt{3})$. (c) The two-fold degenerate band structure of the $\pi$-flux state in the reduced Brillouin zone of an 8-site hexagonal unit cell. leads to a lower energy than the 0-flux state, as already stated above.

Starting from the noninteracting wave function, with a band populated up to the Dirac node at $\varepsilon_{D}=-\sqrt{3} t$ for any of the four flavored fermions, we implement the Gutzwiller projection using VMC sampling. The energy of this wave function, $E=-0.894$ per site, compares remarkably well with that of iPEPS (see Fig. 2), especially considering that no variational parameter was used. Let us also mention that the state (and the ones related by symmetry) shown in Fig. 1(a) has the maximal weight in the variational wave function.

To investigate the physics of this wave function, we have calculated the spin-spin correlation function as a function of distance. The results clearly demonstrate an algebraic decay $\left|\left\langle P_{i j}-1 / 4\right\rangle\right| \sim\left|\mathbf{r}_{i j}\right|^{-\alpha}$, with an exponent $\alpha$ between 3 and 4, as shown in Fig. 6. If one considers the honeycomb lattice as built from zigzag chains, these correlations correspond to even distances along one of the zigzag chains, and the exponent should be compared to that of the dominant correlations with wave vector $\pi / 2$ of a single chain [36]. This exponent is equal to $3 / 2$, a number actually very accurately reproduced by VMC. So color-color correlations decay faster on the honeycomb lattice than on a chain, but still algebraically. This is a rather peculiar situation in view of the standard paradigms: the development of longrange order, as in weakly coupled $\mathrm{SU}(2)$ chains in square geometry, or the spontaneous formation of local singlets and exponentially decaying color-color correlations, as, e.g., in the SU(4) ladder [17].

This Gutzwiller projected $\pi$-flux state is actually a prototypical wave function for a phase that should be called

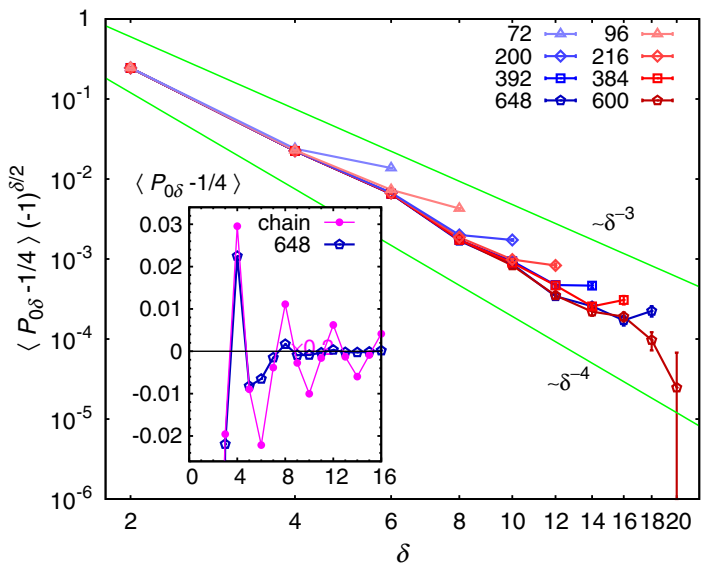

FIG. 6. The algebraic decay of the correlation along a zigzag chain in the honeycomb lattice for the Gutzwiller projected $\pi$-flux state in different clusters. (Every second site is shown; $\delta$ is the distance along the chain.) In the inset, we compare the correlations of the same $\pi$-flux state with the correlations of a 300-site-long, one-dimensional chain. (We projected the onedimensional quarter-filled Fermi sea.) While the periodicity of four is visible in both cases, the correlations decay much faster in the two-dimensional honeycomb lattice. 
algebraic spin-orbital liquid in the present context, in analogy to the algebraic spin liquids that have been discussed in the spin-liquid literature [24,26,27,37]. These states are characterized by the algebraic decay of a number of correlations, with wave vectors corresponding to differences between the Dirac cone loci. In our case, for example, the algebraic spin-orbital correlations are modulated with wave vector $M$ in the standard honeycomb Brillouin zone, which corresponds to the distance between two Dirac cones in the $\pi$-flux state. Another important aspect of this wave function is that it has been shown that it can describe an extended phase in parameter space and not just an unstable fine-tuned point $[26,38]$. On adding perturbations of suitable strength, many different phases can be found in the vicinity of an algebraic spin-orbital liquid [26], making the present model an interesting starting point for further explorations of exotic phases in spin-orbital systems.

\section{CONCLUSIONS}

In conclusion, the results reported in this paper provide very strong evidence that the SU(4) symmetric KugelKhomskii model is a quantum spin-orbital liquid and build a case in favor of an algebraic spin-orbital liquid. Clearly, the present results do not allow one to exclude the possibility that the quantum spin-orbital liquid is of another type, for instance, some kind of resonating valence-bond liquid with resonances between four-site cluster singlets, but our results suggest that the corresponding gap would be quite small. In particular, previous variational studies of highly frustrated magnets have shown that, as good as its energy might be, a fermionic variational wave function might fail to capture the correct low-energy physics. This seems, for instance, to be the case for the SU(2) Heisenberg model on the kagome lattice, for which the variational energy of the algebraic spin-liquid wave function [27] is close to the best numerical estimates [39,40], yet densitymatrix-renormalization-group results have given strong evidence in favor of a gapped $Z_{2}$ spin liquid [39,41]. Actually, the algebraic spin liquid seems to be stable against perturbation of the variational trial state toward a gapped $Z_{2}$ liquid [42]. In the present case, not only has the projected fermionic wave function been tested for its energy, but correlations have also been shown to be in remarkable agreement with ED up to intermediate distances. So we believe that the case for an algebraic spinorbital liquid is strong, but not closed.

In any case, the fact that the ground state is a quantum spin-orbital liquid is quite firmly established. This result is quite interesting in view of the liquid behavior reported recently in $\mathrm{Ba}_{3} \mathrm{CuSb}_{2} \mathrm{O}_{9}$. Among the two scenarios put forward in Ref. [14], i) a random spin singlet stabilized by a disordered static Jahn-Teller distortion, or ii) a dynamic Jahn-Teller effect with fluctuating spins and orbitals, the ground state of the symmetric Kugel-Khomskii model proposed in the present paper is closer to the second one. In particular, there is no sign of glassiness in our calculations since this would imply a configuration with random colors and well-developed local moments, whereas the iPEPS ground state exhibits short-range order with no local moment. However, to make a detailed comparison with experiments, the present model should be extended in several ways. First, some asymmetry between spins and orbitals should be introduced, not only because asymmetry should be present on general grounds (the SU(4) symmetry requires equal hopping amplitudes between the two types of orbitals and neglects the Hund's rule coupling as well as the coupling to phonons), but also in view of the nearest-neighbor spin-spin singlet correlations detected in experiments $[14,43]$. Indeed, in the SU(4) symmetric version of the model, the energy of a pair of neighboring sites can be minimized equally well by a spin singlet or a spin triplet, provided the orbitals form a triplet or a singlet, respectively, and it will take some level of asymmetry to favor spin singlets. In addition, it will be important to take into account the additional magnetic $\mathrm{Cu}$ sites present in the system on top of the honeycomb lattice not only because they are coupled to the spins of the honeycomb lattice, but also because of the disorder they probably induce in the system. These extensions go far beyond the scope of the present paper and are left for future investigation.

Let us also mention that, although it is not chiral, the $\pi$-flux state considered in the present paper can be seen as a natural extension of the chiral states introduced in Ref. [44] in the context of $\mathrm{SU}(\mathrm{N})$ models on the square lattice [45] and recently shown to provide the best fermionic mean-field candidate for the SU(6) Heisenberg model on the honeycomb lattice [46].

Finally, we note that the SU(4) Heisenberg model is a rather accurate effective model for the $1 / N$-filled Mott insulating phase of alkaline-earth metal atoms with $\mathrm{N}$ internal degrees of freedom loaded in an optical lattice $[44,47,48]$. Currently, the main issue in that field is to reach low enough entropies to observe correlations typical of long-range order, but the next step will definitely be to realize exotic quantum states. In that respect, the $N=4$ case on the honeycomb lattice appears to be a very strong candidate.

\section{ACKNOWLEDGMENTS}

The authors thank M. Hermele, V. Gurarie, and M.-F. Yang for clarifying discussions. The ED simulations have been performed on machines of the research platform "Scientific Computing" at the University of Innsbruck, supported by the BMWF, and the iPEPS simulations have been performed on the Brutus cluster at ETH Zurich. We are grateful for the support of the Swiss National Science Foundation, MaNEP, and the Hungarian OTKA Grant No. K73455. 


\section{APPENDIX A: METHODS}

\section{Linear flavor-wave theory}

The LFWT is a method of treating harmonic quantum fluctuations on top of a mean-field (or Hartree) solution based on a site-factorized variational wave function $[49,50]$. The method starts from a Schwinger boson representation of the SU(4) operators with four types of bosons. In a mean-field ground state, each site has a well-defined color. The corresponding boson is assumed to condense, and the resulting Hamiltonian is a bosonic quadratic form. On a nearest-neighbor bond with color $\alpha$ on site $i$ and color $\beta$ on site $j$, the Hamiltonian is given by $\mathcal{H}_{\mathrm{fw}}=$ $A_{i j}^{\dagger} A_{i j}-1$, with $A_{i j}^{\dagger}=b_{\alpha, j}^{\dagger}+b_{\beta, i}$ where $b^{\dagger}$ and $b$ are bosonic operators. In a given mean-field ground state, the LFWT Hamiltonian is the sum of independent Hamiltonians that describe the motion of bosons on the connected clusters spanned by pairs of colors. The zeropoint energy per bond tends to increase with the cluster size and is minimal on a two-site cluster, when the ground-state energy of the Hamiltonian is equal to -1 , i.e., there is no zero-point contribution to the energy. By contrast, larger clusters lead to finite frequencies, and hence to strictly positive contributions to the zero-point energy.

\section{Infinite projected entangled-pair states}

A projected entangled-pair state (PEPS) [51,52], also called a tensor product state, is a variational ansatz where the wave function of a two-dimensional system is efficiently represented by a product of tensors, with one tensor per lattice site. It can be seen as a two-dimensional generalization of matrix product states-the class of variational states underlying the famous density-matrix-renormalization-group method [53]. On the square lattice, each tensor $T_{i j k l}^{p}$ has a physical index $p$ carrying the local Hilbert space of a lattice site with dimension $d$, and four auxiliary bond indices $i, j, k, l$ with dimension $D$ that connect to the four neighboring tensors. Thus, each tensor consists of $d D^{4}$ variational parameters, and, by changing the bond dimension $D$, the accuracy of the ansatz can be systematically controlled. A $D=1$ PEPS simply corresponds to a site-factorized wave function (a product state), and, on increasing $D$, quantum fluctuations can be systematically taken into account.

An infinite PEPS (iPEPS) [54,55] consists of a unit cell of $L_{x} \times L_{y}=N_{T}$ tensors that is periodically repeated in the lattice to represent a wave function in the thermodynamic limit. We use the iPEPS method developed for the square lattice described in Refs. $[20,54,56]$ to simulate the model on the honeycomb lattice by mapping it onto a brickwall lattice as illustrated in Fig. 7(a). The bond dimension of the auxiliary bonds connecting two sites that do not directly interact (dotted lines) can be chosen as $D=1$. This ansatz is equivalent to an iPEPS with only three auxiliary indices on the honeycomb lattice. The
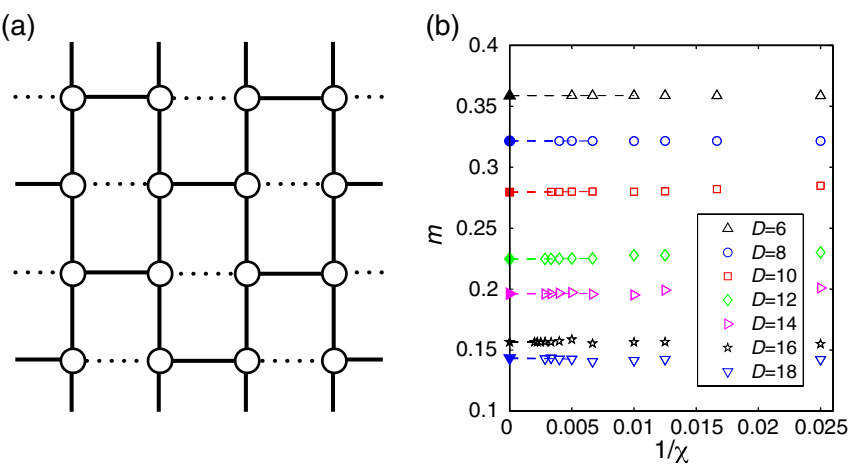

FIG. 7. (a) The honeycomb lattice is mapped to a square lattice with brickwall structure. There is no Hamiltonian term between sites connected by a dotted line. A square-lattice iPEPS is used for this lattice, where we choose the bond dimension along the dotted lines as $D=1$. (b) Local ordered moment as a function of inverse $\chi$, which controls the accuracy of the contraction of the iPEPS. The values of $m$ for different bond dimensions $D$ depend only slightly on $\chi$.

advantage of this mapping is that the codes developed for the square lattice require only minor modifications to simulate models on the honeycomb lattice.

Describing the iPEPS method in full detail is beyond the scope of this paper, and we therefore mention only the most important technicalities, with corresponding references and details, on the simulation parameters in the following discussion. For an introduction to PEPS and iPEPS, we refer to Refs. [52,56].

The optimization of the tensors (i.e., finding the best variational parameters) is done through imaginary time evolution, first with the so-called simple update (see Refs. [56,57]), which is equivalent to the time-evolving block-decimation method in one dimension [58,59]. The solution is then used as an initial state for an imaginary time evolution using the full update [54,56], which is computationally more expensive, but more accurate than the simple update, since the full wave function is taken into account for the truncation of a bond index. We use a second-order Trotter-Suzuki decomposition with time steps down to $d \tau=10^{-3}$. For large values of $D$, a larger time step $d \tau=10^{-2}$ is used, where the estimated Trotter error is small compared to symbol sizes [e.g., below $0.5 \%$ for the ordered moment $m(2)]$.

Expectation values of observables can be computed by contracting the tensor network, i.e., by computing the trace of the product of all tensors. For the approximate contraction of the iPEPS, we use the corner-transfer-matrix method described in Refs. [56,60,61]. The accuracy of this contraction can be controlled by the so-called boundary dimension $\chi$, where we use values up to $\chi=500$ (typically, up to $\chi=$ 350 ) for large $D$. Observables like the energy and the ordered moment are extrapolated in $\chi$, with an extrapolation error being small compared to symbol sizes. An example is shown in Fig. 7(b) for the ordered moment $m$ (2). 
To increase the efficiency of the method, we have implemented a $\mathbb{Z}_{q}$ symmetry, a discrete subgroup of SU(4), in the tensors (see Refs. [62,63]), leading to tensors that have a block structure (similar to a block diagonal matrix) and to a considerable reduction of the computational cost.

Since iPEPS is an ansatz for an infinite system, symmetries such as $\mathrm{SU}(4)$ or translational symmetry may be spontaneously broken. In order to test different types of translational symmetry breaking, we have compared the variational energies obtained with different unit cell sizes. We have found two competing low-energy states with unit cell sizes $2 \times 2$ and $4 \times 4$, shown in Fig. 3, which have similar energies for large bond dimension. We note that broken symmetries can be an artifact of a finite bond dimension $D$, and that the symmetry can be restored if $D$ is sufficiently large. In other words, a classical or a lowentanglement solution (small $D$ ) might exhibit order, but this order can be destroyed by quantum fluctuations that are systematically included on increasing $D$. Therefore, it is important to study order parameters as a function of $D$ to verify if they are finite in the large- $D$ limit.

\section{Exact diagonalization}

We have performed exact diagonalizations of the Hamiltonian (1) for selected finite-size samples of up to $N=24$ sites. The energies of the samples with 8,14,18 and 24 sites are reported with star symbols in Fig. 2. Note that only the samples with 8 and 24 sites can form a SU(4) singlet ground state, which explains the relatively high energy per site for the samples with 14 and 18 sites. Note also that, due to computational limitations, we were able to calculate only the energy and eigenfunction of the ground state in the completely symmetric representation of the spatial symmetry group of the $N=24$ sample. (The Hilbert space in this symmetry sector contains 4008417658 states, including a cyclic color-permutation symmetry.) Since this sector is the absolute ground state for the $N=8$ sample, we expect this sector to host the ground state for $N=24$ as well.

\section{Fermionic variational Monte Carlo method}

The variational wave function for the algebraic spinorbital liquid is a Gutzwiller projected noninteracting Fermi sea at quarter filling,

$$
|\Psi\rangle=\sum_{\{j\}} \prod_{\alpha=1}^{4} w_{\left\{j^{\alpha}\right\}}\left|j_{1}^{\alpha} j_{2}^{\alpha} \ldots j_{N / 4}^{\alpha}\right\rangle,
$$

where $j_{l}^{\alpha}$ denotes the position of the $l$ th fermion with color $\alpha$, and the summation is over all $N ! /[(N / 4) !]^{4}$ possible distributions of the fermions so that each site is single occupied (i.e., $\left\{j^{\alpha}\right\} \cap\left\{j^{\beta}\right\}$ is an empty set for $\alpha \neq \beta$ ). The weight of each configuration is given by the Slater determinant

$$
w_{\left\{j^{\alpha}\right\}}=\left|\begin{array}{cccc}
\xi_{1}\left(j_{1}^{\alpha}\right) & \xi_{2}\left(j_{1}^{\alpha}\right) & \ldots & \xi_{N / 4}\left(j_{1}^{\alpha}\right) \\
\vdots & \vdots & \ddots & \vdots \\
\xi_{1}\left(j_{N / 4}^{\alpha}\right) & \xi_{2}\left(j_{N / 4}^{\alpha}\right) & \ldots & \xi_{N / 4}\left(j_{N / 4}^{\alpha}\right)
\end{array}\right|
$$

where $\xi_{k}(j)$ is the amplitude of the fermion at site $j$ in the $k$ th eigenfunction of the hopping Hamiltonian

$$
\mathcal{H}_{f}=-\sum_{\langle i, j\rangle} \sum_{\alpha=1}^{4}\left(\chi_{i, j} f_{j, \alpha}^{\dagger} f_{i, \alpha}+\text { H.c. }\right) .
$$

The expectation values of operators with this variational wave function are evaluated by a Monte Carlo sampling $[29,30]$. The variational wave function with Majorana fermions is described in detail in Ref. [8].

The different trial states correspond to different choices of the $\chi_{i, j}$ hopping parameters. In the $\pi$-flux state, $\left|\chi_{i, j}\right|=$ 1 , and the phases are chosen so that an electron hopping around the hexagon picks up a minus sign, $\prod_{k=1}^{6} \chi_{i_{k}, i_{k+1}}=$ -1 , where the product is over the bonds of a hexagon. Many possible choices of phases lead to such a minus sign. Here, we choose real hopping amplitudes, where every hexagon has a single bond with a $\chi_{i j}=-1$, while the rest of the bonds have +1 , as shown in Fig. 5(a). Furthermore, we allow for antiperiodic boundary conditions when a degeneracy of quarter-filled Fermi sea needs to be removed for a given cluster.

We have considered two families of finite-size clusters with the full $D_{6}$ symmetry of the honeycomb lattice: (i) clusters with $N=2(2 n)^{2}$ sites defined by the lattice vectors $\mathbf{g}_{1}=(\sqrt{3}, 0) n$ and $\mathbf{g}_{2}=(\sqrt{3} / 2,3 / 2) n$, where $n$ is an integer ( $N=72,200,392$, and 648 site clusters) and the bond length is set to unity; and (ii) clusters with $N=6(2 n)^{2}$ sites with $\mathbf{g}_{1}=(3 \sqrt{3} / 2,3 / 2) n$ and $\mathbf{g}_{2}=$ $(0,3) n,(N=24,96,216,384,600)$.

We use Monte Carlo sampling of the projected wave function $|\Psi\rangle$ to evaluate physical quantities. The elementary step is the exchange of two randomly chosen fermions with different colors. To speed up the convergence, we used importance sampling, with acceptance ratios defined according to the Metropolis algorithm: The new configuration is always accepted if its weight $w_{\text {new }}$ is higher than the weight $w_{\text {old }}$ of the old configuration, but, for $w_{\text {new }}<w_{\text {old }}$, the configuration is accepted only with a probability $w_{\text {new }} / w_{\text {old }}$. The configurations are thus represented with a probability $p_{\{j\}}$ proportional to their weight in the wave function,

$$
p_{\{j\}} \propto\left|w_{\{j\}}\right|^{2},
$$

where $w_{\{j\}}=\prod_{\alpha=1}^{4} w_{\left\{j^{\alpha}\right\}}$ denotes the coefficient of $|\{j\}\rangle=$ $\otimes_{\alpha=1}^{4}\left|j_{1}^{\alpha} j_{2}^{\alpha} \ldots j_{N / 4}^{\alpha}\right\rangle$ in the projected fermionic state $|\Psi\rangle$ [see Eq. (A2)]. We set the number of elementary steps between two measurements large enough to avoid autocorrelation effects. See Table I for details. We have also 
TABLE I. $\tau_{\text {a.c. }}$ autocorrelation times for the two sitecorrelation functions compared to the number of elementary steps between two measurements $(\Delta n)$.

\begin{tabular}{rrrrc}
\hline \hline $\mathrm{N}$ & $\tau_{\text {a.c. }}$ & \multicolumn{1}{c}{$\Delta n$} & Ratio & Number of measurements \\
\hline 24 & 22 & 1000 & 45.5 & $10^{7}$ \\
72 & 150 & 1000 & 6.7 & $10^{7}$ \\
96 & 260 & 2000 & 7.7 & $10^{7}$ \\
200 & 970 & 5000 & 5.1 & $2 \times 10^{6}$ \\
216 & 1080 & 5000 & 4.6 & $2 \times 10^{6}$ \\
384 & 2920 & 20000 & 6.8 & $2 \times 10^{6}$ \\
392 & 3340 & 20000 & 6.0 & $2 \times 10^{6}$ \\
600 & 7080 & 40000 & 5.6 & $10^{6}$ \\
648 & 8100 & 40000 & 4.9 & $10^{6}$ \\
\hline \hline
\end{tabular}

performed a binning analysis as a further test for the independence of the measurements. The statistical error for different bin sizes did not show any change, verifying once more that the sampling distances were large enough. Furthermore, for each system, we have made several (5-10) runs with randomly chosen starting configurations to independently verify the error bars obtained from the binning analysis.

We have measured diagonal and off-diagonal operators. The spin-spin correlation function, the average of the offdiagonal $P_{k, l}$, can be expressed using the diagonal $n_{k}^{\beta} n_{l}^{\beta}$ operator (where $n_{k}^{\beta}$ is the occupation number on site $k$ for the fermion of color $\beta$ ) as

$$
\left\langle P_{k, l}\right\rangle=20\left\langle n_{k}^{\beta} n_{l}^{\beta}\right\rangle-1,
$$

supposing that the ground state is a singlet wave functionas is the case when the hopping Hamiltonian is independent of the colors. The measurement of the diagonal $\left\langle n_{k}^{\beta} n_{l}^{\beta}\right\rangle$ correlation functions is quite simple using importance sampling,

$$
\left\langle n_{k}^{\beta} n_{l}^{\beta}\right\rangle_{\mathrm{MC}}=\frac{N\left(\{k, l\} \subset\left\{j^{\beta}\right\}\right)}{N_{\mathrm{MC}}},
$$

where $N\left(\{k, l\} \subset\left\{j^{\beta}\right\}\right)$ denotes the number of times both $k$ and $l$ sites are occupied with $\beta$ fermion among the $N_{\mathrm{MC}}$ measured configurations.

With a little more effort, one can directly calculate the off-diagonal $\left\langle P_{k, l}\right\rangle$ correlation functions as well. Using the fermionic representation for the $P_{k, l}$ exchange operator, the convenient form that follows the convention of the fermion ordering in the wave function is given as

$$
P_{k, l}=\sum_{\alpha \beta} S_{\alpha}^{\beta}(k) S_{\beta}^{\alpha}(l)=-\sum_{\alpha \beta} f_{\alpha}^{\dagger}(k) f_{\beta}^{\dagger}(l) f_{\beta}(k) f_{\alpha}(l)
$$

In this case, one follows the same importance sampling as before, although the measurement itself is more complicated:

$$
\begin{aligned}
\left\langle P_{k, l}\right\rangle & =\frac{\sum_{\{j\},\{j\}} \bar{w}_{\{j\}} w_{\{j\}}\left\langle\{j\}\left|P_{k, l}\right|\{\tilde{j}\}\right\rangle}{\sum_{\{j\}}\left|w_{\{j\}}\right|^{2}} \\
& =\frac{\sum_{\{j\}}\left|w_{\{j\}}\right|^{2}{\frac{w}{\left\{j^{\prime}\right\}}}_{\left.w_{j j\}}\right\}} s_{k, l}(\{j\})}{\sum_{\{j\}}\left|w_{\{j\}}\right|^{2}} \\
& =\frac{1}{N_{\mathrm{MC}}} \sum_{\{j\}_{\mathrm{MC}}} \frac{w_{\left\{j^{\prime}\right\}}}{w_{\{j\}}} s_{k, l}(\{j\}),
\end{aligned}
$$

where $\left\{j^{\prime}\right\}$ is the configuration that leads to $\{j\}$ by exchanging the color of fermions on sites $k$ and $l$, and the sum in the last equation is over the measured configuration $\{j\}_{\mathrm{MC}}$. Following Eq. (A7), the sign $s_{k, l}(\{j\})$ is 1 if the colors of fermions are the same on sites $k$ and $l$ in the configuration $\{j\}$, and -1 if the colors are different. We have explicitly verified that the Eq. (A5) holds.

Similarly, one can calculate $\left\langle P_{i j} P_{k l}\right\rangle$ as well, here for each $\{j\}$ configuration $|\{j\}\rangle=P_{i j} P_{k l}\left|\left\{j^{\prime}\right\}\right\rangle$. For the sign that one should take $s_{k, l}(\{j\}) s_{i, j}(\{j\})$, here we have assumed that the sites $i, j, k$, and $l$ are all different.

[1] J. Schlappa, K. Wohlfeld, K. J. Zhou, M. Mourigal, M. W. Haverkort, V. N. Strocov, L. Hozoi, C. Monney, S. Nishimoto, S. Singh et al., Spin-Orbital Separation in the Quasi-One-Dimensional Mott Insulator $\mathrm{Sr}_{2} \mathrm{CuO}_{3}$, Nature (London) 485, 82 (2012).

[2] J. van den Brink, Z. Nussinov, and A.M. Oleś, in Introduction to Frustrated Magnetism, edited by $\mathrm{C}$. Lacroix, P. Mendels, and F. Mila, Springer Series in Solid-State Sciences, Vol. 164 (Springer, Berlin, 2011), pp. 629-670, ISBN 978-3-642-10589-0.

[3] S. Ishihara, M. Yamanaka, and N. Nagaosa, Orbital Liquid in Perovskite Transition-Metal Oxides, Phys. Rev. B 56, 686 (1997).

[4] L.F. Feiner, A. M. Oleś, and J. Zaanen, Quantum Melting of Magnetic Order Due to Orbital Fluctuations, Phys. Rev. Lett. 78, 2799 (1997).

[5] Y. Q. Li, M. Ma, D. N. Shi, and F. C. Zhang, SU(4) Theory for Spin Systems with Orbital Degeneracy, Phys. Rev. Lett. 81, 3527 (1998).

[6] G. Khaliullin and S. Maekawa, Orbital Liquid in ThreeDimensional Mott Insulator: $\mathrm{LaTiO}_{3}$, Phys. Rev. Lett. 85, 3950 (2000).

[7] F. Vernay, K. Penc, P. Fazekas, and F. Mila, Orbital Degeneracy as a Source of Frustration in $\mathrm{LiNiO}_{2}$, Phys. Rev. B 70, 014428 (2004).

[8] F. Wang and A. Vishwanath, $\mathrm{Z}_{2}$ Spin-Orbital Liquid State in the Square Lattice Kugel-Khomskii Model, Phys. Rev. B 80, 064413 (2009).

[9] J. Chaloupka and A. M. Oleś, Spin-Orbital Resonating Valence Bond Liquid on a Triangular Lattice: Evidence from Finite-Cluster Diagonalization, Phys. Rev. B 83, 094406 (2011).

[10] Y. Kitaoka, T. Kobayashi, A. Kōda, H. Wakabayashi, Y. Niino, H. Yamakage, S. Taguchi, K. Amaya, K. Yamaura, M. Takano et al., Orbital Frustration and Resonating 
Valence Bond State in the Spin-1/2 Triangular Lattice $\mathrm{LiNiO}_{2}$, J. Phys. Soc. Jpn. 67, 3703 (1998).

[11] F. Reynaud, D. Mertz, F. Celestini, J. Debierre, A. M. Ghorayeb, P. Simon, A. Stepanov, J. Voiron, and C. Delmas, Orbital Frustration at the Origin of the Magnetic Behavior in $\mathrm{LiNiO}_{2}$, Phys. Rev. Lett. 86, 3638 (2001).

[12] V. Fritsch, J. Hemberger, N. Büttgen, E. Scheidt, H. Krug von Nidda, A. Loidl, and V. Tsurkan, Spin and Orbital Frustration in $\mathrm{MnSc}_{2} \mathrm{~S}_{4}$ and $\mathrm{FeSc}_{2} \mathrm{~S}_{4}$, Phys. Rev. Lett. 92, 116401 (2004).

[13] M. V. Mostovoy and D. I. Khomskii, Orbital Ordering in Frustrated Jahn-Teller Systems with $90^{\circ}$ Exchange, Phys. Rev. Lett. 89, 227203 (2002).

[14] S. Nakatsuji, K. Kuga, K. Kimura, R. Satake, N. Katayama, E. Nishibori, H. Sawa, R. Ishii, M. Hagiwara, F. Bridges et al., Spin-Orbital Short-Range Order on a HoneycombBased Lattice, Science 336, 559 (2012).

[15] K. I. Kugel' and D. I. Khomskiǔ, The Jahn-Teller Effect and Magnetism: Transition Metal Compounds, Sov. Phys. Usp. 25, 231 (1982).

[16] M. van den Bossche, F. Zhang, and F. Mila, Plaquette Ground State in the Two-Dimensional SU(4) Spin-Orbital Model, Eur. Phys. J. B 17, 367 (2000).

[17] M. van den Bossche, P. Azaria, P. Lecheminant, and F. Mila, Spontaneous Plaquette Formation in the SU(4) SpinOrbital Ladder, Phys. Rev. Lett. 86, 4124 (2001).

[18] H. Hung, Y. Wang, and C. Wu, Quantum Magnetism in Ultracold Alkali and Alkaline-Earth Fermion Systems with Symplectic Symmetry, Phys. Rev. B 84, 054406 (2011).

[19] E. Szirmai and M. Lewenstein, Exotic Magnetic Orders for High-Spin Ultracold Fermions, Europhys. Lett. 93, 66005 (2011).

[20] P. Corboz, A. M. Läuchli, K. Penc, M. Troyer, and F. Mila, Simultaneous Dimerization and SU(4) Symmetry Breaking of 4-Color Fermions on the Square Lattice, Phys. Rev. Lett. 107, 215301 (2011).

[21] P. Corboz, K. Penc, F. Mila, and A. M. Läuchli, Simplex Solids in SU(N) Heisenberg Models on the Kagome and Checkerboard Lattices, Phys. Rev. B 86, 041106 (2012).

[22] The variational energies (per site) of the $\pi$-flux, Majorana 0 -flux, 0-flux, and Majorana $\pi$-flux states in the 96-site cluster are $-0.895,-0.822,-0.763$, and -0.755 , respectively.

[23] LFWT leads to a very low energy. This is not a concern since the method is not variational, but it is an indication that, in the present case, higher orders are likely to be important.

[24] X. Wen, Quantum Orders and Symmetric Spin Liquids, Phys. Rev. B 65, 165113 (2002).

[25] O. I. Motrunich, Variational Study of Triangular Lattice Spin-1/2 Model with Ring Exchanges and Spin Liquid State in $\kappa-(\mathrm{ET})_{2} \mathrm{Cu}_{2}(\mathrm{CN})_{3}$, Phys. Rev. B 72, 045105 (2005).

[26] M. Hermele, T. Senthil, and M. P. A. Fisher, Algebraic Spin Liquid as the Mother of Many Competing Orders, Phys. Rev. B 72, 104404 (2005).

[27] Y. Ran, M. Hermele, P. A. Lee, and X. Wen, ProjectedWave-Function Study of the Spin-1/2 Heisenberg Model on the Kagomé Lattice, Phys. Rev. Lett. 98, 117205 (2007).

[28] A. Paramekanti and J. B. Marston, $S U(N)$ Quantum Spin Models: A Variational Wavefunction Study, J. Phys. Condens. Matter 19, 125215 (2007).
[29] H. Yokoyama and H. Shiba, Variational Monte-Carlo Studies of Hubbard Model. I, J. Phys. Soc. Jpn. 56, 1490 (1987).

[30] C. Gros, Physics of Projected Wavefunctions, Ann. Phys. (N.Y.) 189, 53 (1989).

[31] I. Affleck and J.B. Marston, Large-n Limit of the Heisenberg-Hubbard Model: Implications for High- $T_{c}$ Superconductors, Phys. Rev. B 37, 3774 (1988).

[32] T. A. Kaplan, P. Horsch, and P. Fulde, Close Relation between Localized-Electron Magnetism and the Paramagnetic Wave Function of Completely Itinerant Electrons, Phys. Rev. Lett. 49, 889 (1982).

[33] M. Hermele, SU(2) Gauge Theory of the Hubbard Model and Application to the Honeycomb Lattice, Phys. Rev. B 76, 035125 (2007).

[34] A. F. Albuquerque, D. Schwandt, B. Hetényi, S. Capponi, M. Mambrini, and A. M. Läuchli, Phase Diagram of a Frustrated Quantum Antiferromagnet on the Honeycomb Lattice: Magnetic Order versus Valence-Bond Crystal Formation, Phys. Rev. B 84, 024406 (2011).

[35] B. K. Clark, D. A. Abanin, and S. L. Sondhi, Nature of the Spin Liquid State of the Hubbard Model on a Honeycomb Lattice, Phys. Rev. Lett. 107, 087204 (2011).

[36] B. Frischmuth, F. Mila, and M. Troyer, Thermodynamics of the One-Dimensional SU(4) Symmetric Spin-Orbital Model, Phys. Rev. Lett. 82, 835 (1999).

[37] F.F. Assaad, Phase Diagram of the Half-Filled TwoDimensional $S U(N)$ Hubbard-Heisenberg Model: A Quantum Monte Carlo Study, Phys. Rev. B 71, 075103 (2005).

[38] W. Rantner and X.-G. Wen, Electron Spectral Function and Algebraic Spin Liquid for the Normal State of Underdoped High $T_{c}$ Superconductors, Phys. Rev. Lett. 86, 3871 (2001).

[39] S. Yan, D. A. Huse, and S. R. White, Spin-Liquid Ground State of the $S=1 / 2$ Kagome Heisenberg Antiferromagnet, Science 332, 1173 (2011).

[40] A. M. Läuchli, J. Sudan, and E. S. Sørensen, Ground-State Energy and Spin Gap of Spin-1/2 Kagomé-Heisenberg Antiferromagnetic Clusters: Large-Scale Exact Diagonalization Results, Phys. Rev. B 83, 212401 (2011).

[41] S. Depenbrock, I.P. McCulloch, and U. Schollwöck, Nature of the Spin-Liquid Ground State of the $S=1 / 2$ Heisenberg Model on the Kagome Lattice, Phys. Rev. Lett. 109, 067201 (2012).

[42] Y. Iqbal, F. Becca, and D. Poilblanc, Projected Wave Function Study of $\mathrm{Z}_{2}$ Spin Liquids on the Kagome Lattice for the Spin-1/2 Quantum Heisenberg Antiferromagnet, Phys. Rev. B 84, 020407 (2011).

[43] J. A. Quilliam, F. Bert, E. Kermarrec, C. Payen, C. GuillotDeudon, P. Bonville, C. Baines, H. Luetkens, and P. Mendels, Singlet Ground State of the Quantum Antiferromagnet $\mathrm{Ba}_{3} \mathrm{CuSb}_{2} \mathrm{O}_{9}$, Phys. Rev. Lett. 109, 117203 (2012).

[44] M. Hermele, V. Gurarie, and A. M. Rey, Mott Insulators of Ultracold Fermionic Alkaline Earth Atoms: Underconstrained Magnetism and Chiral Spin Liquid, Phys. Rev. Lett. 103, 135301 (2009).

[45] M. Hermele (private communication).

[46] G. Szirmai, E. Szirmai, A. Zamora, and M. Lewenstein, Gauge Fields Emerging from Time-Reversal Symmetry 
Breaking for Spin-5/2 Fermions in a Honeycomb Lattice, Phys. Rev. A 84, 011611 (2011).

[47] M. A. Cazalilla, A. F. Ho, and M. Ueda, Ultracold Gases of Ytterbium: Ferromagnetism and Mott States in an SU(6) Fermi System, New J. Phys. 11, 103033 (2009).

[48] A. V. Gorshkov, M. Hermele, V. Gurarie, C. Xu, P. S. Julienne, J. Ye, P. Zoller, E. Demler, M. D. Lukin, and A. M. Rey, Two-Orbital SU(N) Magnetism with Ultracold Alkaline-Earth Atoms, Nat. Phys. 6, 289 (2010).

[49] N. Papanicolaou, Pseudospin Approach for Planar Ferromagnets, Nucl. Phys. B240, 281 (1984).

[50] A. Joshi, M. Ma, F. Mila, D. N. Shi, and F. C. Zhang, Elementary Excitations in Magnetically Ordered Systems with Orbital Degeneracy, Phys. Rev. B 60, 6584 (1999).

[51] F. Verstraete and J. I. Cirac, Renormalization Algorithms for Quantum-Many Body Systems in Two and Higher Dimensions, arXiv:cond-mat/0407066.

[52] F. Verstraete, V. Murg, and J. I. Cirac, Matrix Product States, Projected Entangled Pair States, and Variational Renormalization Group Methods for Quantum Spin Systems, Adv. Phys. 57, 143 (2008).

[53] S. R. White, Density Matrix Formulation for Quantum Renormalization Groups, Phys. Rev. Lett. 69, 2863 (1992).

[54] J. Jordan, R. Orús, G. Vidal, F. Verstraete, and J. I. Cirac, Classical Simulation of Infinite-Size Quantum Lattice Systems in Two Spatial Dimensions, Phys. Rev. Lett. 101, 250602 (2008).
[55] P. Corboz, S. R. White, G. Vidal, and M. Troyer, Stripes in the Two-Dimensional $t$-J Model with Infinite Projected Entangled-Pair States, Phys. Rev. B 84, 041108 (2011).

[56] P. Corboz, R. Orús, B. Bauer, and G. Vidal, Simulation of Strongly Correlated Fermions in Two Spatial Dimensions with Fermionic Projected Entangled-Pair States, Phys. Rev. B 81, 165104 (2010).

[57] H. Jiang, Z. Weng, and T. Xiang, Accurate Determination of Tensor Network State of Quantum Lattice Models in Two Dimensions, Phys. Rev. Lett. 101, 090603 (2008).

[58] G. Vidal, Efficient Classical Simulation of Slightly Entangled Quantum Computations, Phys. Rev. Lett. 91, 147902 (2003).

[59] R. Orús and G. Vidal, Infinite Time-Evolving Block Decimation Algorithm beyond Unitary Evolution, Phys. Rev. B 78, 155117 (2008).

[60] T. Nishino and K. Okunishi, Corner Transfer Matrix Renormalization Group Method, J. Phys. Soc. Jpn. 65, 891 (1996).

[61] R. Orús and G. Vidal, Simulation of Two-Dimensional Quantum Systems on an Infinite Lattice Revisited: Corner Transfer Matrix for Tensor Contraction, Phys. Rev. B 80, 094403 (2009).

[62] S. Singh, R. N. C. Pfeifer, and G. Vidal, Tensor Network Decompositions in the Presence of a Global Symmetry, Phys. Rev. A 82, 050301 (2010).

[63] B. Bauer, P. Corboz, R. Orús, and M. Troyer, Implementing Global Abelian Symmetries in Projected Entangled-Pair State Algorithms, Phys. Rev. B 83, 125106 (2011). 